\title{
Three-dimensional edge waves in plates
}

\author{
By V. ZERNOV ${ }^{1} \dagger$, J. KAPLUnOV ${ }^{2}$ \\ ${ }^{1}$ Dept. Engineering, Science and The Built Environment, \\ London South Bank University, 103 Borough Road, London, SE1 OAA \\ 2 Dept. of Mathematical Sciences, Brunel University, \\ Kingston Lane, Uxbridge, UB8 3PH
}

The paper describes the propagation of three-dimensional symmetric waves localised near the traction free edge of a semi-infinite elastic plate with either traction free or fixed faces. For both types of boundary conditions, we present a variational proof of the existence of the low order edge waves. In addition, for a plate with traction free faces and zero Poisson ratio, the fundamental edge wave is described by a simple explicit formula, and the first order edge wave is proved to exist. Qualitative variational predictions are compared with numerical results, which are obtained using expansions in three-dimensional Rayleigh-Lamb and shear modes. It is also demonstrated numerically that whatever non-zero Poisson ratio in a plate with traction free faces, the eigenfrequencies related to the first order wave are complex valued.

Keywords: edge wave, elastic plate, variation, eigen-spectrum, Rayleigh-Lamb

\section{Introduction}

Edge waves in plates are well known in two-dimensional approximate structural theories, which describe long-wave low-frequency motions. In particular, in the theory of plate extension, that is the generalised plane stress (Love 1944), the edge wave is a natural analogue of the Rayleigh wave. The first example of a dispersive edge wave was derived in Konenkov (1960) using the classical theory of plate bending. In anisotropic thin plates, the existence and uniqueness of flexural edge waves were studied in $\mathrm{Fu}$ (2003). Counterparts of bending and extensional edge waves also appear in the two-dimensional shell theory (e. g. Kaplunov et al. 2000).

In the three-dimensional context, there is a simple analytical solution for a plate with mixed boundary conditions (Kaplunov et al. 2005). The resulting infinite family of edge waves is closely related to the Rayleigh wave propagating along the free edge. Related trapped modes in elastic structures have been recently studied in Förster et al. (2006), Postnova et al. (2006) and Adams et al. (2007).

In some sense, this paper generalises the results on complex eigenvalues of a semi-infinite strip (Pagneux 2006 and Zernov et al. 2006), which coincide with the cut-off frequencies of the first order edge wave. We study three-dimensional symmetric edge waves in a plate with either traction free or fixed faces. For the former, the only example of a closed form expression that we could find corresponds to the

$\dagger$ Author for correspondence. 
fundamental edge wave in the case of zero Poisson ratio (section 4). This generalises the edge wave arising in the two-dimensional theory of plate extension. For all other cases the three-dimensional symmetric edge wave can be expressed as infinite series in Rayleigh-Lamb and shear modes, which we investigate numerically. The computed spectrum generally determines elastic waves with non-localised components, related to complex eigenvalues. For the real eigenvalues associated with the edge waves under consideration, we adopt a variational approach, which involves minimisation of the appropriate functionals along with the estimation of continuous spectra (Roitberg et al. 1998).

Three types of edge waves are considered, the fundamental, the first order edge wave in a plate with traction free faces (the latter only for the zero Poisson ratio) and the first (lowest) order edge wave in a plate with fixed faces. To estimate continuous spectra we analyse dispersion relations. In particular, for the fundamental wave in a plate with traction free faces we arrive at the estimate involving the speed of the Rayleigh wave. The Rayleigh wave also appears in the test functions introduced to minimise the variational functional in section 8 . In section 7 we also develop a numerical minimisation technique that relies on finite dimensional linear spans of Rayleigh-Lamb and shear modes.

\section{Statement of the problem}

Let us consider a semi-infinite elastic plate of unit half-thickness, occupying the three-dimensional region $\mathcal{H}=\left\{\left(x_{1}, x_{2}, x_{3}\right) \in \mathbb{R}^{3}: x_{1}>0, x_{2} \in J\right\}, J:=(-1,1)$ (see Figure 1). Let its edge and faces be denoted by $\partial \mathcal{H}_{e}=\left\{\left(x_{1}, x_{2}, x_{3}\right) \in \mathbb{R}^{3}\right.$ : $\left.x_{1}=0,-1 \leqslant x_{2} \leqslant 1\right\}$ and $\partial \mathcal{H}_{ \pm}=\left\{\left(x_{1}, x_{2}, x_{3}\right) \in \mathbb{R}^{3}: x_{1} \geqslant 0, x_{2}= \pm 1\right\}$ respectively, and let $\boldsymbol{n}=\left(n_{1}, n_{2}, n_{3}\right)$ be the unit outward normal to the plate surface $\partial \mathcal{H}=\partial \mathcal{H}_{e} \bigcup \partial \mathcal{H}_{ \pm}$.

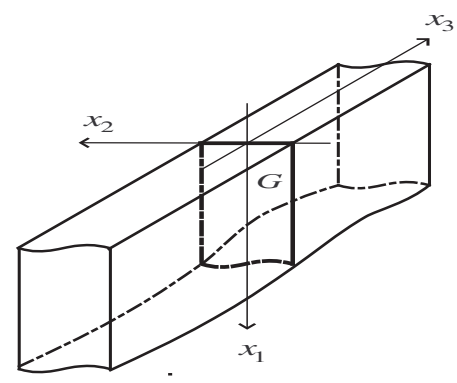

Figure 1. Configuration of three-dimensional plate.

Small harmonic vibrations of an isotropic homogeneous plate are governed by the classical equation of linear elasticity. Introducing the displacement vector $\boldsymbol{U}=$ $\left(U_{1}, U_{2}, U_{3}\right)$, it can be presented in an operator form as

$$
\mathcal{L} \boldsymbol{U}=\lambda \boldsymbol{U}
$$


where the spectral parameter is $\lambda=k^{2}$, with

$$
k=\frac{\omega}{c_{t}}
$$

and the operator $\mathcal{L}$ is defined by

$$
\mathcal{L} \boldsymbol{U}=-\triangle \boldsymbol{U}-\left(\chi^{2}-1\right) \operatorname{grad} \operatorname{div} \boldsymbol{U}
$$

with

$$
\chi^{2}=\frac{c_{l}^{2}}{c_{t}^{2}}=\frac{2-2 \nu}{1-2 \nu} .
$$

Above, $\omega$ is the circular frequency and the longitudinal and transverse waves speeds are given by

$$
c_{l}=\sqrt{\frac{E(1-\nu)}{\rho(1+\nu)(1-2 \nu)}}, \quad c_{t}=\sqrt{\frac{E}{2 \rho(1+\nu)}},
$$

and the constants $\rho>0, E>0, \nu \geqslant 0$ denote respectively the volume density, Young's modulus and Poisson ratio. The factor $\exp (-i \omega t)$ is implied but omitted everywhere.

The traction free boundary conditions take the form

$$
\left.\boldsymbol{T}\right|_{\partial \mathcal{H}}=\mathbf{0} .
$$

whereas for a plate with a free edge and fixed faces the boundary conditions are

$$
\left.\boldsymbol{T}\right|_{\partial \mathcal{H}_{e}}=\mathbf{0},\left.\quad \boldsymbol{U}\right|_{\partial \mathcal{H}_{ \pm}}=\mathbf{0},
$$

here the stress components $\sigma_{i j}$, as expressed in terms of the displacements, being

$$
\sigma_{i j}=\frac{E}{2(1+\nu)}\left(\left(\chi^{2}-2\right) \operatorname{div} \boldsymbol{U} \delta_{i j}+\left(U_{i, j}+U_{j, i}\right)\right), \quad i, j=1,2,3,
$$

with the surface traction vector $\boldsymbol{T}=\left(T_{1}, T_{2}, T_{3}\right)$ given by

$$
T_{i}=\frac{2(1+\nu)}{E} \sigma_{i j} n_{j}, \quad i=1,2,3 .
$$

We are interested in the edge waves that propagate along the $x_{3}$-axis and decay as $x_{1} \rightarrow+\infty$. The corresponding displacement field can be expressed as

$$
\boldsymbol{U}\left(x_{1}, x_{2}, x_{3}\right)=\boldsymbol{u}\left(x_{1}, x_{2}\right) e^{\mathrm{i} \xi x_{3}}, \xi \in R .
$$

By substituting (2.10) into the equations of motion (2.3) we reduce the original three-dimensional problem on $\mathcal{H}$ to a two-dimensional one on the plate cross-section $G:=\left\{\left(x_{1}, x_{2}\right) \in \mathbb{R}^{2}: x_{1} \geqslant 0,-1 \leqslant x_{2} \leqslant 1\right\}$. This can be written as

$$
\mathcal{L}_{\xi} \boldsymbol{u}=k^{2} \boldsymbol{u},
$$


with

$$
\mathcal{L}_{\xi} \boldsymbol{u}=\triangle_{\xi} \boldsymbol{u}+\left(\chi^{2}-2\right) \operatorname{grad}_{\xi} \operatorname{div}_{\xi} \boldsymbol{u}
$$

where

$$
\begin{gathered}
\triangle_{\xi} \boldsymbol{u}=\boldsymbol{u}_{, 11}+\boldsymbol{u}_{, 22}-\xi^{2} \boldsymbol{u}, \quad \operatorname{div}_{\xi} \boldsymbol{u}=u_{1,1}+u_{2,2}+\mathrm{i} \xi u_{3}, \\
\operatorname{grad}_{\xi} v=\left(\begin{array}{lll}
v_{, 1} & v_{, 2} & \mathrm{i} \xi v
\end{array}\right)^{T},
\end{gathered}
$$

and

$$
\boldsymbol{u} \in W_{2}^{2}\left(G, \mathbb{C}^{3}\right), \quad v\left(x_{1}, x_{2}\right) \in W_{2}^{2}(G, \mathbb{C}) .
$$

Here and everywhere below $W_{2}^{i}$ denote Sobolev spaces.

The domain $D\left(\mathcal{L}_{\xi}\right)$ of the operator $\mathcal{L}_{\xi}$ is given by

$$
D\left(\mathcal{L}_{\xi}\right):=\left\{\boldsymbol{u} \in W_{2}^{2}\left(G, \mathbb{C}^{3}\right):\left.\boldsymbol{T}_{\xi}\right|_{\partial G}=\mathbf{0}\right\},
$$

where the traction vector $\boldsymbol{T}_{\xi}$ is the counterpart of the vector (2.9), corresponding to the transformation (2.10).

Let us consider the quadratic form

$$
\begin{aligned}
& a_{\xi}[\boldsymbol{u}, \boldsymbol{u}]=\frac{1}{2} \sum_{i, j=1}^{2}\left\|u_{i, j}+u_{j, i}\right\|_{L_{2}(G)}^{2}+ \\
& \quad+\sum_{i=1}^{2}\left\|u_{3, i}+\mathrm{i} \xi u_{i}\right\|_{L_{2}(G)}^{2}+2\left\|\xi^{2} u_{3}\right\|_{L_{2}(G)}^{2}+\left(\chi^{2}-2\right)\left\|\operatorname{div}_{\xi} \boldsymbol{u}\right\|_{L_{2}(G)}^{2},
\end{aligned}
$$

on the domain $d\left[a_{\xi}\right]=W_{2}^{1}\left(G, \mathbb{C}^{3}\right)$. For some positive constant $C$ the following inequalities hold

$$
C^{-1}\|\boldsymbol{u}\|_{W_{2}^{1}}^{2} \leqslant a_{\xi}[\boldsymbol{u}, \boldsymbol{u}]+\|\boldsymbol{u}\|_{L_{2}}^{2} \leqslant C\|\boldsymbol{u}\|_{W_{2}^{1}}^{2},
$$

for all $\boldsymbol{u} \in d\left[a_{\xi}\right]$. The right inequality in (2.15) is obvious, whereas the left one is nontrivial and it is an analogue of the Korn's inequality (Gobert 1962). According to $(2.15)$ the form $a_{\xi}$ is closed and semi-bounded from below, therefore it induces unique self-adjoint operator $\overline{\mathcal{L}}_{\xi}$ with some domain $D\left(\overline{\mathcal{L}}_{\xi}\right) \subset d\left[a_{\xi}\right]$ (see Birman \& Solomjak 1986). On the other hand it is easy to see that $\left(\mathcal{L}_{\xi} \boldsymbol{u}, \boldsymbol{u}\right)=a_{\xi}[\boldsymbol{u}, \boldsymbol{u}]$ for any $\boldsymbol{u} \in D\left(\mathcal{L}_{\xi}\right)$ and that $D\left(\mathcal{L}_{\xi}\right)$ is dense in $d\left[a_{\xi}\right]$ with respect to the norm $\sqrt{a_{\xi}[\boldsymbol{u}, \boldsymbol{u}]}+$ $\|\boldsymbol{u}\|_{L_{2}}$. Therefore $\overline{\mathcal{L}}_{\xi}$ is the Friedrichs extension of $\mathcal{L}_{\xi}$ (Birman \& Solomjak 1986). This leads to a two-dimensional eigenvalue problem for the self-adjoint operator $\overline{\mathcal{L}}_{\xi}$.

Since the plate motions can be decomposed into symmetric and anti-symmetric with respect to its mid-plane, the operator $\overline{\mathcal{L}}_{\xi}$ has the following invariant projectors

$$
P_{i}\left(\begin{array}{l}
u_{1}\left(x_{1}, x_{2}\right) \\
u_{2}\left(x_{1}, x_{2}\right) \\
u_{3}\left(x_{1}, x_{2}\right)
\end{array}\right)=\frac{1}{2}\left(\begin{array}{l}
u_{1}\left(x_{1}, x_{2}\right)-(-1)^{i} u_{1}\left(x_{1},-x_{2}\right) \\
u_{2}\left(x_{1}, x_{2}\right)+(-1)^{i} u_{2}\left(x_{1},-x_{2}\right) \\
u_{3}\left(x_{1}, x_{2}\right)-(-1)^{i} u_{3}\left(x_{1},-x_{2}\right)
\end{array}\right), \quad i=1,2 .
$$

onto the invariant subspaces $H_{s}=P_{1} D\left(\overline{\mathcal{L}}_{\xi}\right)$ and $H_{a}=P_{2} D\left(\overline{\mathcal{L}}_{\xi}\right)$. In this paper we restrict ourselves to the analysis of the symmetric operator $A_{\xi}=\left.\overline{\mathcal{L}}_{\xi}\right|_{H_{s}}$. 


\section{The Rayleigh-Lamb and shear modes}

The original equations allow the separation of variables

$$
\boldsymbol{U}\left(x_{1}, x_{2}, x_{3}\right)=\boldsymbol{V}\left(x_{2}\right) e^{\mathrm{i}\left(\alpha x_{1}+\xi x_{3}\right)}
$$

which results in dispersion relations for harmonic waves which propagate in an infinite plate.

In particular, in the case of traction free faces $\left(\boldsymbol{T}=\mathbf{0}\right.$ at $\left.x_{2}= \pm 1\right)$ we have the Rayleigh-Lamb equation (see e. g. Miklowitz 1978)

$$
\Phi(p, k):=\left(2 p^{2}-k^{2}\right)^{2} \cosh \gamma \sinh \delta-4 p^{2} \gamma \delta \sinh \gamma \cosh \delta=0,
$$

with

$$
\gamma=\sqrt{p^{2}-\frac{k^{2}}{\chi^{2}}}, \delta=\sqrt{p^{2}-k^{2}}, p=\sqrt{\alpha^{2}+\xi^{2}},
$$

for symmetric waves and the equation

$$
\sinh \delta=0
$$

for shear waves.

The real solutions of the Rayleigh-Lamb equation (3.2), which correspond to propagating modes (3.1), may be arranged in the set

$$
\mathcal{K}^{R L}:=\left\{k_{0}^{R L}(\alpha, \xi) \leqslant k_{1}^{R L}(\alpha, \xi) \leqslant \ldots \leqslant k_{n}^{R L}(\alpha, \xi) \leqslant \ldots\right\}, \quad \alpha \in[0,+\infty) .
$$

Similarly, for real solutions of (3.4) we can write

$$
\mathcal{K}^{S H}:=\left\{k_{0}^{S H}(\alpha, \xi) \leqslant k_{1}^{S H}(\alpha, \xi) \leqslant \ldots \leqslant k_{n}^{S H}(\alpha, \xi) \leqslant \ldots\right\}, \quad \alpha \in[0,+\infty) .
$$

Below, we also use the union $\mathcal{K}:=\mathcal{K}^{R L} \cup \mathcal{K}^{S H}$ of the propagating modes, rearranged in ascending order

$$
\mathcal{K}:=\left\{k_{0}(\alpha, \xi) \leqslant k_{1}(\alpha, \xi) \leqslant \ldots \leqslant k_{n}(\alpha, \xi) \leqslant \ldots\right\}, \quad \alpha \in[0,+\infty) .
$$

The set (3.7) defines the dispersion curves for the elastic waves that propagate in a three-dimensional plate.

Using the solutions of the dispersion relation (3.2), the displacement in the $\mathrm{n}$-th Rayleigh-Lamb mode may be written as

$$
\boldsymbol{V}_{n}^{R L}\left(x_{2}\right)=\left(\begin{array}{c}
\delta_{n} B_{n} \alpha_{n} \cosh \left(\delta_{n} x_{2}\right)+\mathrm{i} p_{n} \alpha_{n} \cosh \left(\gamma_{n} x_{2}\right) \\
-\mathrm{i} p_{n}^{2} B_{n} \sinh \left(\delta_{n} x_{2}\right)+p_{n} \gamma_{n} \sinh \left(\gamma_{n} x_{2}\right) \\
\xi \delta_{n} B_{n} \cosh \left(\delta_{n} x_{2}\right)+\mathrm{i} \xi p_{n} \cosh \left(\gamma_{n} x_{2}\right)
\end{array}\right)
$$

with

$$
B_{n}=\frac{\left(2 p_{n}^{2}-k^{2}\right) \cosh \left(\gamma_{n}\right)}{2 \mathrm{i} p_{n} \delta_{n} \cosh \left(\delta_{n}\right)} .
$$

For the shear modes, we have

$$
\boldsymbol{V}_{n}^{S H}\left(x_{2}\right)=\left(\begin{array}{c}
i \xi \cosh \left(\delta_{n} x_{2}\right) \\
0 \\
-\mathrm{i} \alpha_{n} \cosh \left(\delta_{n} x_{2}\right)
\end{array}\right)
$$


The stress components associated with the displacements in the formulae (3.1), (3.2) and (3.4) may be written as

$$
\left(\begin{array}{l}
\sigma_{11} \\
\sigma_{12} \\
\sigma_{13}
\end{array}\right)=\boldsymbol{S}_{n}\left(x_{2}\right) e^{\mathrm{i}\left(\alpha_{n} x_{1}\right)}
$$

For the Rayleigh-Lamb modes the traction vector $\boldsymbol{S}_{n}\left(x_{2}\right)$ has the following form

$$
\boldsymbol{S}_{n}^{R L}\left(x_{2}\right)=\left(\begin{array}{c}
2 \mathrm{i} \alpha_{n}^{2} \delta_{n} B_{n} \cosh \left(\delta_{n} x_{2}\right)+p_{n}\left(2 \xi^{2}-2 \gamma_{n}^{2}-k^{2}\right) \cosh \left(\gamma_{n} x_{2}\right) \\
B_{n} \alpha_{n}\left(2 p_{n}^{2}-k^{2}\right) \sinh \left(\delta_{n} x_{2}\right)+2 \mathrm{i} p_{n} \alpha_{n} \gamma_{n} \sinh \left(\gamma_{n} x_{2}\right) \\
2 \mathrm{i} \xi \alpha_{n} \delta_{n} B_{n} \cosh \left(\delta_{n} x_{2}\right)-2 p_{n} \alpha_{n} \xi \cosh \left(\gamma_{n} x_{2}\right)
\end{array}\right)
$$

and for the shear modes it is given by

$$
\boldsymbol{S}_{n}^{S H}\left(x_{2}\right)=\left(\begin{array}{c}
-2 \alpha_{n} \xi \cosh \left(\delta_{n} x_{2}\right) \\
\mathrm{i} \xi \delta_{n} \sinh \left(\delta_{n} x_{2}\right) \\
\left(\alpha_{n}^{2}-\xi^{2}\right) \cosh \left(\delta_{n} x_{2}\right)
\end{array}\right)
$$

The edge waves under consideration may be expanded in an infinite series of the Rayleigh-Lamb and shear modes (3.8) and (3.10) to satisfy the boundary conditions on a traction free edge. In general, the coefficients in the series cannot be found explicitly. One exception is presented in the next section.

\section{Explicit solution for the fundamental edge wave in case of zero Poisson ratio}

Apparently, the only example of an explicit solution for a three-dimensional edge wave can be constructed for $\nu=0$. In the latter case, the dispersion relations (3.2) and (3.4) have two imaginary roots $\alpha=\alpha_{0}^{R L}=\mathrm{i} \sqrt{\xi^{2}-k^{2} / 2}$ and $\alpha=\alpha_{0}^{S H}=$ $\mathrm{i} \sqrt{\xi^{2}-k^{2}}$, which correspond to the fundamental Rayleigh-Lamb and shear mode respectively. At the traction free edge $x_{1}=0$ we consider a linear combination of the corresponding stresses (3.12) and (3.13)

$$
C_{1} \boldsymbol{S}_{0}^{S H}+C_{2} \boldsymbol{S}_{0}^{R L}=C_{1}\left(\begin{array}{c}
2 \mathrm{i} \xi \sqrt{\xi^{2}-k^{2}} \\
0 \\
2 \xi^{2}-k^{2}
\end{array}\right)+C_{2} k\left(\begin{array}{c}
\mathrm{i} \sqrt{\xi^{2}-k^{2} / 2} \\
0 \\
\xi
\end{array}\right)=\left(\begin{array}{l}
0 \\
0 \\
0
\end{array}\right)
$$

where $C_{1}$ and $C_{2}$ are arbitrary constants. The homogeneous system (4.1) has a non-trivial solution provided we have

$$
\left(2 \xi^{2}-k^{2}\right)^{2}-4 \xi^{2} \sqrt{\xi^{2}-k^{2}} \sqrt{\xi^{2}-k^{2} / 2}=0 .
$$

It is worth noting that equation (4.2) is a Rayleigh equation (A 4) for $\nu=0$. It is satisfied when $k=\xi \sqrt{3-\sqrt{5}}$. For this value of $k$ the corresponding wave numbers are $\alpha_{0}^{R L}=i \xi \sqrt{(\sqrt{5}-1) / 2}$ and $\alpha_{0}^{S H}=i \xi \sqrt{\sqrt{5}-2}$, and the displacements in the edge waves can be written as

$$
\boldsymbol{u}\left(x_{1}, x_{2}\right)=\boldsymbol{r}_{0}\left(x_{1}, \xi\right),
$$


where $\boldsymbol{r}_{\nu}\left(x_{1}, \xi\right)$ is the Rayleigh wave (A 2) at the Poisson ration $\nu$.

The relations (4.2) and (4.3) determine a wave that is uniformly distributed along plate thickness and coincides with the Rayleigh wave propagating along the surface of an elastic half-plane $x_{1}>0,-\infty<x_{3}<\infty$. The observed wave may be regarded as a fundamental edge wave, since as the wave number $\xi$ vanishes its frequency tends to zero. The result generalises the edge wave which arises in the two-dimensional approximate theory of plate extension (e. g. Kaplunov et al. 2000).

The existence of an explicit solution for $\nu=0$ is closely related to the additional internal symmetry of the operator $A_{\xi}$, which was introduced in section 2 . The latter assures that both fundamental are orthogonal to all other Rayleigh-Lamb and shear modes, that is, the following conditions are satisfied

$$
\begin{aligned}
& \left(\boldsymbol{S}_{0}^{R L}, \boldsymbol{S}_{j}^{R L}\right)_{L_{2}\left(J, \mathbb{C}^{3}\right)}=0, \quad\left(\boldsymbol{S}_{0}^{R L}, \boldsymbol{S}_{j}^{S H}\right)_{L_{2}\left(J, \mathbb{C}^{3}\right)}=0, \quad j \neq 0, \\
& \left(\boldsymbol{S}_{0}^{S H}, \boldsymbol{S}_{j}^{R L}\right)_{L_{2}\left(J, \mathbb{C}^{3}\right)}=0, \quad\left(\boldsymbol{S}_{0}^{S H}, \boldsymbol{S}_{j}^{S H}\right)_{L_{2}\left(J, \mathbb{C}^{3}\right)}=0, \quad j \neq 0 .
\end{aligned}
$$

This can be easily verified by integration (see Zernov 2006). Consider an edge wave with the stress field $\boldsymbol{S}\left(x_{1}, x_{2}\right)$, its expansion into the series of the Rayleigh-Lamb and shear modes may be written as

$$
\boldsymbol{S}\left(x_{1}, x_{2}\right)=\sum_{j=0}^{\infty} C_{j}^{R L} \boldsymbol{S}_{j}^{R L}\left(x_{2}\right) e^{\mathrm{i} \alpha_{j}^{R L} x_{1}}+\sum_{j=0}^{\infty} C_{j}^{S H} \boldsymbol{S}_{j}^{S H}\left(x_{2}\right) e^{\mathrm{i} \alpha_{j}^{S H} x_{1}},
$$

where $C_{j}^{R L}$ and $C_{j}^{S H}$ are some unknown coefficients. The traction free boundary conditions on the edge have the form

$$
\left\|\boldsymbol{S}\left(0, x_{2}\right)\right\|_{L_{2}(J)}=0
$$

Substituting (4.5) into (4.6) and making use of the orthogonality conditions (4.4) we obtain

$$
\begin{aligned}
\left\|C_{0}^{R L} \boldsymbol{S}_{0}^{R L}+C_{0}^{S H} \boldsymbol{S}_{0}^{S H}\right\|_{L_{2}(J)} & =0, \\
\left\|\sum_{j=1}^{\infty} C_{j}^{R L} \boldsymbol{S}_{j}^{R L}+\sum_{j=1}^{\infty} C_{j}^{S H} \boldsymbol{S}_{j}^{S H}\right\|_{L_{2}(J)} & =0 .
\end{aligned}
$$

Relations (4.7) and (4.8) imply that the families of the fundamental and all other modes do not interact on the edge and may be studied separately. As it was shown above, a linear combination of the $\boldsymbol{S}_{0}^{R L}$ and $\boldsymbol{S}_{0}^{S H}$ gives an explicit solution for the fundamental edge wave. All other edge waves do not contain any fundamental components, i.e. $C_{0}^{R L}=C_{0}^{S H}=0$. This point will be discussed further in section 7 .

\section{The variational approach}

When the Poisson ratio is non-zero the analysis of the fundamental symmetric edge wave requires a more delicate handling. In this section, we develop a variational technique, which can also be extended to the higher order edge waves.

Consider the spectrum $\sigma\left(A_{\xi}\right)$ of the operator $A_{\xi}$, which was introduced in section 2. Since $A_{\xi}$ is positive definite, $\sigma\left(A_{\xi}\right) \subset[0,+\infty)$. In addition, $\sigma\left(A_{\xi}\right)$ contains 
a continuous interval $\sigma_{c}\left(A_{\xi}\right)$, which is associated with propagating Rayleigh-Lamb (3.2) and shear modes (3.4). For the latter we have

$$
\sigma_{c}\left(A_{\xi}\right)=\left[\Upsilon^{2}\left(A_{\xi}\right),+\infty\right),
$$

with

$$
\Upsilon\left(A_{\xi}\right):=\min _{\alpha \in[0,+\infty)} k_{0}(\alpha, \xi)
$$

(see Birman \& Solomjak 1986, Roitberg et al. 1998)

The edge wave is related to the discrete eigenvalues of the operator $A_{\xi}$. To investigate the existence of a discrete spectrum located to the left of the continuous interval $\sigma_{c}\left(A_{\xi}\right)$ we adapt the variational formula

$$
\min \sigma\left(A_{\xi}\right)=\inf \left\{\frac{a_{\xi}[\boldsymbol{u}, \boldsymbol{u}]}{(\boldsymbol{u}, \boldsymbol{u})_{L_{2}(G)}}, \boldsymbol{u} \in P_{1} d\left[a_{\xi}\right]\right\} .
$$

This means that, we need to specify a test function $\boldsymbol{u}_{t} \in P_{1} d\left[a_{\xi}\right]$, such that

$$
\frac{a_{\xi}\left[\boldsymbol{u}^{t}, \boldsymbol{u}^{t}\right]}{\left(\boldsymbol{u}^{t}, \boldsymbol{u}^{t}\right)_{L_{2}(G)}}<\Upsilon^{2}\left(A_{\xi}\right)
$$

(e. g. Davies 1995, Birman \& Solomjak 1986)

\section{Existence of a fundamental edge wave for an arbitrary Poisson ratio.}

The variational technique described in the previous section relies on an estimate $\Upsilon\left(A_{\xi}\right)$ of the lower bound of the continuous spectrum $\sigma_{c}\left(A_{\xi}\right)$. We have for the latter

$$
\Upsilon\left(A_{\xi}\right)=\min \left(\Upsilon^{R L}\left(A_{\xi}\right), \Upsilon^{S H}\left(A_{\xi}\right)\right),
$$

where we use the notations

$$
\Upsilon^{R L}\left(A_{\xi}\right)=\min _{\alpha \in[0,+\infty)} k_{0}^{R L}(\alpha, \xi), \quad \Upsilon^{S H}\left(A_{\xi}\right)=\min _{\alpha \in[0,+\infty)} k_{0}^{S H}(\alpha, \xi) .
$$

The shear dispersion equation (3.4) has a simple solution

$$
\Upsilon^{S H}\left(A_{\xi}\right)=\xi
$$

It follows from (3.2) that the equality

$$
k_{0}^{R L}(\alpha, \xi)=k_{0}^{R L}\left(\sqrt{\alpha^{2}+\xi^{2}}, 0\right)
$$

is valid for any $\alpha$ and $\xi$. Therefore in the case of the Rayleigh-Lamb dispersion relation we have

$$
\Upsilon^{R L}\left(A_{\xi}\right)=\min _{\alpha \in[0,+\infty)} k_{0}^{R L}\left(\sqrt{\alpha^{2}+\xi^{2}}, 0\right)=\min _{\alpha \geqslant \xi} k_{0}^{R L}(\alpha, 0) .
$$


Next, by substituting $\xi=0$ into (3.2), in the vicinity of $\alpha=0$, we obtain the following asymptotic expression

$$
k_{0}^{R L}(\alpha, 0)=2 \sqrt{\frac{\chi^{2}-1}{\chi^{2}}} \alpha+O\left(\alpha^{3}\right), \quad \alpha \rightarrow 0,
$$

where we have

$$
2 \sqrt{\frac{\chi^{2}-1}{\chi^{2}}} \geqslant \sqrt{2}>1, \quad \nu \in[0,1 / 2]
$$

For large values of $\alpha$, the asymptotic behaviour is given by

$$
k_{0}^{R L}(\alpha, 0)=c_{R} \alpha+O\left(e^{-2 \Lambda_{T} \alpha}\right), \quad \alpha \rightarrow \infty .
$$

In addition, at $\xi=0$, the secular equation (3.2) has no roots along the straight line $k=c_{R} \alpha$. In fact, we have

$\Phi\left(\alpha, c_{R} \alpha\right)=\alpha^{4}\left(\left(1+\Lambda_{T}^{2}\right)^{2} \cosh \left(\alpha \Lambda_{L}\right) \sinh \left(\alpha \Lambda_{T}\right)-4 \Lambda_{T} \Lambda_{L} \sinh \left(\alpha \Lambda_{L}\right) \cosh \left(\alpha \Lambda_{T}\right)\right)$.

Since $c_{R}$ is a solution of (A 4$)$, we can write

$$
\left|\Phi\left(\alpha, c_{R} \alpha\right)\right|=\left|4 \alpha^{4} \Lambda_{T} \Lambda_{L} \sinh \left(\alpha \Lambda_{L}\right) \cosh \left(\alpha \Lambda_{T}\right)\right|\left|\frac{\tanh \left(\alpha \Lambda_{T}\right)}{\tanh \left(\alpha \Lambda_{L}\right)}-1\right|>0, \alpha>0 .
$$

Expression (6.6) implies that for small $\alpha, k_{0}^{R L}(\alpha, 0)>c_{R} \alpha$ and formula (6.9) guarantees that for any $\alpha, k_{0}^{R L}(\alpha, 0) \neq c_{R} \alpha$. The continuity of $k_{0}^{R L}(\alpha, 0)$ implies that

$$
k_{0}^{R L}(\alpha, 0)>c_{R} \alpha, \quad \alpha>0 .
$$

Therefore we finally obtain

$$
\Upsilon^{R L}\left(A_{\xi}\right)=\min _{\alpha \geqslant \xi} k_{0}^{R L}(\alpha, 0)>\min _{\alpha \geqslant \xi} c_{R} \alpha=c_{R} \xi
$$

Combined with (6.3) this yields the estimate

$$
\Upsilon\left(A_{\xi}\right)=\min \left(\Upsilon^{R L}\left(A_{\xi}\right), \Upsilon^{S H}\left(A_{\xi}\right)\right)>c_{R} \xi
$$

which suggests choosing the classical Rayleigh wave (A 2) for a test function. With this choice the variational formula (5.3) becomes

$$
\min \sigma\left(A_{\xi}\right) \leqslant \frac{a_{\xi}\left[\boldsymbol{r}_{\nu}, \boldsymbol{r}_{\nu}\right]}{\left(\boldsymbol{r}_{\nu}, \boldsymbol{r}_{\nu}\right)}=\frac{\left(A_{\xi} \boldsymbol{r}_{\nu}, \boldsymbol{r}_{\nu}\right)+\int_{\partial G} \boldsymbol{T}_{\xi}\left(\boldsymbol{r}_{\nu}\right) \overline{\boldsymbol{r}_{\nu}} d g}{\left(\boldsymbol{r}_{\nu}, \boldsymbol{r}_{\nu}\right)} .
$$

The integral in (6.13) is zero since it represents the total energy flux through $\partial G$. By inserting the identity $A_{\xi} \boldsymbol{r}_{\nu}=c_{R}^{2} \xi^{2} \boldsymbol{r}_{\nu}$ into (6.13), we finally arrive at the inequalities

$$
\min \sigma\left(A_{\xi}\right) \leqslant c_{R}^{2} \xi^{2}<\Upsilon^{2}\left(A_{\xi}\right), \quad \xi>0 .
$$

These prove the existence of the fundamental edge wave for $\nu<1 / 2$. 


\section{The higher order edge waves}

It has been shown previously (Pagneux 2006, Zernov et al. 2006) that in a semiinfinite elastic strip the eigenvalues are as a rule complex. For this reason, it can be expected that in the three-dimensional case, the eigenvalues corresponding to the higher order edge waves are complex too. Numerical experiments have confirmed that indeed, in general, the edge waves contain not only decaying components but also components propagating in the $x_{1}$-direction. As a consequence, the vibrations are damped and the corresponding eigenfrequency is complex.

However, we can show that when $\nu=0$, there exists a higher order edge wave with a real eigenvalue, i.e. an edge wave that has no propagating component. Indeed, as mentioned above, in this case there exists an additional internal symmetry, resulting in the orthogonality conditions (4.4). As a result, similarly to the two-dimensional case (see Roitberg et al. 1998) the operator $A_{\xi}$ has an invariant subspace.

Let us introduce the notation

$$
P_{0}\left(\begin{array}{l}
u_{1}\left(x_{1}, x_{2}\right) \\
u_{2}\left(x_{1}, x_{2}\right) \\
u_{3}\left(x_{1}, x_{2}\right)
\end{array}\right)=\frac{1}{2}\left(\begin{array}{c}
\int_{-1}^{1} u_{1}\left(x_{1}, x_{2}\right) d x_{2} \\
0 \\
\int_{-1}^{1} u_{3}\left(x_{1}, x_{2}\right) d x_{2}
\end{array}\right), \boldsymbol{u} \in H_{s} .
$$

Then $P_{0}$ is an orthogonal projection from $H_{s}$ onto the subspace

$$
H_{s 0}=\left\{\boldsymbol{u} \in H_{s}: u_{1}\left(x_{1}, x_{2}\right)=u_{1}\left(x_{1}\right), u_{2}=0, u_{3}\left(x_{1}, x_{2}\right)=u_{3}\left(x_{1}\right)\right\},
$$

and the orthogonal complement of $H_{s 0}$ in $H_{s}$ is

$$
H_{s 1}=\left\{\boldsymbol{u} \in H_{s}: \int_{-1}^{1} u_{1}\left(x_{1}, x_{2}\right) d x_{2}=0, \int_{-1}^{1} u_{3}\left(x_{1}, x_{2}\right) d x_{2}=0\right\} .
$$

Elementary calculations show that $H_{s 0}$ and $H_{s 1}$ are a pair of invariant subspaces of the operator $A_{\xi}$. Below we denote the corresponding operators by $A_{\xi 0}=\left.A_{\xi}\right|_{H_{s 0}}$ and $A_{\xi 1}=\left.A_{\xi}\right|_{H_{s 1}}$ respectively. For the operator $A_{\xi 1}$, we have

$$
\Upsilon\left(A_{\xi 1}\right)=\min _{\alpha \in[0, \infty)} k_{1}(\alpha, \xi)>=\min _{\alpha \in[0, \infty)} k_{0}(\alpha, \xi)=\Upsilon\left(A_{\xi}\right),
$$

i. e. the continuous spectrum of the operator $A_{\xi 1}$ is to the right of $\Upsilon\left(A_{\xi}\right)$.

Next, let us we minimise the variational functional (5.3) on the set of the Rayleigh-Lamb and shear modes. The test function composed of a large number of these modes should provide a good approximation to an edge wave. Let us start by defining an Euclidian space $L_{N, M}$ formed by $N$ Rayleigh-Lamb modes and $M$ shear modes (see section 3),

$$
L_{N, M}=\left\{\boldsymbol{u}\left(x_{1}, x_{2}\right)=\sum_{i=1}^{N+M} C_{i} \mathcal{V}_{i}\left(x_{2}\right) e^{i \beta_{i} x_{1}}, C_{i} \in \mathbb{C}\right\}
$$

where we have

$$
\begin{gathered}
\beta_{i}=\alpha_{i}^{R L}, \quad \mathcal{V}_{i}=\boldsymbol{V}_{i}^{R L}, \quad \mathcal{S}_{i}=\boldsymbol{S}_{i}^{R L}, \quad i=1 . . N \\
\beta_{i}=\alpha_{i-N}^{S H}, \quad \mathcal{V}_{i}=\boldsymbol{V}_{i-N}^{S H}, \quad \mathcal{S}_{i}=\boldsymbol{S}_{i-N}^{S H}, \quad i=N+1 . . M+N .
\end{gathered}
$$


Note that $L_{N, M} \subset H_{s 1}$ since the modes $\alpha_{0}^{R L}$ and $\alpha_{0}^{S H}$ do not belong to the space. On the $L_{N, M}$ the variational functional (5.3) takes the form

$$
\begin{array}{r}
\Theta(\boldsymbol{u}, k):=\frac{a_{\xi}[\boldsymbol{u}, \boldsymbol{u}]}{(\boldsymbol{u}, \boldsymbol{u})_{L_{2}(G)}}=k^{2}+\frac{1}{\|\boldsymbol{u}\|^{2}} \int_{-1}^{1} \boldsymbol{T}_{\xi}(\boldsymbol{u}) \overline{\boldsymbol{u}} d x_{2}= \\
=k^{2}+\frac{\sum_{i, j=1}^{N+M} C_{i} \overline{C_{j}}\left(\mathcal{S}_{i}, \mathcal{U}_{j}\right)}{\sum_{i, j=1}^{N+M} C_{i} \overline{C_{j}}\left(\mathcal{U}_{i}, \mathcal{U}_{j}\right)\left(i \overline{\beta_{j}}-i \beta_{i}\right)^{-1}},
\end{array}
$$

where $C_{i}$ are arbitrary constants.

Let us then specify the matrices $Q$ and $R$ with the components

$$
Q_{i j}=\left(\mathcal{S}_{j}, \mathcal{U}_{i}\right)_{L_{2}\left(J, \mathbb{C}^{3}\right)}, \quad R_{i j}=\frac{\left(\mathcal{U}_{j}, \mathcal{U}_{i}\right)_{L_{2}\left(J, \mathbb{C}^{3}\right)}}{i \overline{\beta_{i}}-i \beta_{j}}, \quad i, j=1 . . N+M,
$$

and rewrite the functional (7.7) as

$$
\Theta_{1}(\boldsymbol{C}, k):=\Theta(\boldsymbol{u}, k)=k^{2}+\frac{(Q \boldsymbol{C}, \boldsymbol{C})}{(R \boldsymbol{C}, \boldsymbol{C})},
$$

where the vector $C$ is

$$
\boldsymbol{C}=\left(C_{1}, C_{2}, \ldots, C_{N+M}\right) .
$$

The operator corresponding to matrix $R$ is positive definite since for $k<\Upsilon\left(A_{\xi 1}\right)$, we have $(R \boldsymbol{C}, \boldsymbol{C})=\|\boldsymbol{u}\|_{L_{2}\left(G, \mathbb{C}^{3}\right)}^{2}>0$. Therefore, there exists its unique positive definite square root $R^{\frac{1}{2}}$. In terms of the latter, the variational functional (7.9) becomes

$$
\Theta_{1}(\boldsymbol{C}, k)=k^{2}+\frac{(Q \boldsymbol{C}, \boldsymbol{C})}{\left(R^{\frac{1}{2}} \boldsymbol{C}, R^{\frac{1}{2}} \boldsymbol{C}\right)}=k^{2}+\frac{\left(R^{-\frac{1}{2}} Q R^{-\frac{1}{2}} \boldsymbol{V}, \boldsymbol{V}\right)}{(\boldsymbol{V}, \boldsymbol{V})}=\Theta_{2}(\boldsymbol{V}, k) .
$$

Since the inverse operator $\left(R^{\frac{1}{2}}\right)^{-1}$ exists, the vectors $\boldsymbol{V}=R^{\frac{1}{2}} \boldsymbol{C}, \boldsymbol{C} \in \mathbb{C}^{N+M}$ occupy the whole space $\mathbb{C}^{N+M}$. As a result, we may minimise the functional $\Theta_{2}$ instead of $\Theta_{1}$, i. e. we can write

$$
\min _{\boldsymbol{C} \in \mathbb{C}^{N+M}} \Theta_{1}(\boldsymbol{C}, k)=\min _{\boldsymbol{V} \in \mathbb{C}^{N+M}} \Theta_{2}(\boldsymbol{V}, k) .
$$

It is well known (e. g. Birman \& Solomjak (1986)) that the minimum of the fraction in $\Theta_{2}$ coincides with the minimum of $\sigma\left(R^{-\frac{1}{2}} Q R^{-\frac{1}{2}}\right)$ which consists of a finite number of points on the real axis. Therefore, we have

$$
\min _{\boldsymbol{V} \in \mathbb{C}^{N+M}} \Theta_{2}(\boldsymbol{V}, k)=k^{2}+\min \sigma\left(R^{-\frac{1}{2}} Q R^{-\frac{1}{2}}\right) .
$$

It is clear that we can write

$$
Q R^{-1} \boldsymbol{Y}=\lambda \boldsymbol{Y}
$$

with

$$
\boldsymbol{Y}=R^{\frac{1}{2}} \boldsymbol{X}
$$


where $\lambda$ and $\boldsymbol{X}$ denote an eigenfunction and eigenvector of the operator $R^{-\frac{1}{2}} Q R^{-\frac{1}{2}}$, respectively. Since the operator $R^{\frac{1}{2}}$ is an isomorphism $\mathbb{C}^{N+M} \rightarrow \mathbb{C}^{N+M}$, the formula (7.14) implies $\sigma\left(R^{-\frac{1}{2}} Q R^{-\frac{1}{2}}\right)=\sigma\left(Q R^{-1}\right)$. Thus we arrive at a formula for the numerical evaluation of eigenfrequencies,

$$
k_{T}^{2}\left(A_{\xi 1}\right):=\min _{k<\Upsilon\left(A_{\xi 1}\right)} \min _{\boldsymbol{u} \in L_{N, M}} \Theta(\boldsymbol{u}, k)=\min _{k<\Upsilon\left(A_{\xi 1}\right)}\left(k^{2}+\min \sigma\left(Q R^{-1}\right)\right) .
$$

Figure 2 a) illustrates the proposed approach for $N=20$ and $M=20$. The curve $\Upsilon\left(A_{\xi 1}\right)$ is computed numerically using the formulae (3.2), (3.4) and (5.2). The figure demonstrates that the second order edge wave exists in the interval $\xi \in[0,1.1585)$.

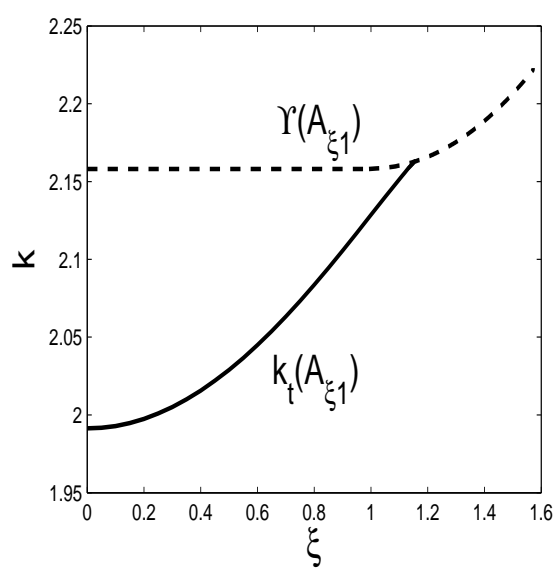

a)

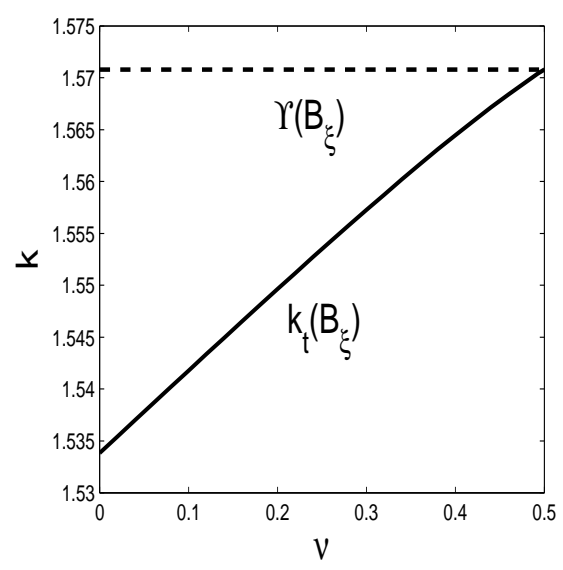

b)

Figure 2. Numerical evaluation of test functions a) the first order edge wave in a plate with traction free faces $(\nu=0) \mathrm{b})$ the first order edge wave in a plate with fixed faces $(\xi=0)$.

\section{A plate with fixed faces}

Let us consider symmetric motion of a plate with fixed faces. In this case, the corresponding related self-adjoint operator $B_{\xi}$ takes the same differential form as the operator $A_{\xi}$, from section 1 and $D\left(B_{\xi}\right) \subset d\left[a_{\xi}\right]$ where

$$
d\left[a_{\xi}\right]:=\left\{\boldsymbol{u} \in W_{2}^{1}\left(G, \mathbb{C}^{3}\right):\left.\boldsymbol{u}\right|_{\partial G_{ \pm}}=\mathbf{0}\right\} .
$$

The dispersion relations, analogous to (3.2) and (3.4), are

$$
\Psi(p, k):=p^{2} \cosh \gamma \frac{\sinh \delta}{\delta}-\gamma \sinh \gamma \cosh \delta=0,
$$

and

$$
\cosh \delta=0
$$

where $p, \gamma$ and $\delta$ are the same as in (3.3). By contrast with the case of traction free faces, these dispersion relations do not support fundamental modes (see e. g. 
Kaplunov et al. 1998 and references therein). Everywhere below in this section the superscript "0" refers to the lowest harmonics.

The dispersion relation (8.3) leads to an explicit estimate of the continuous spectrum

$$
\Upsilon^{S H}\left(B_{\xi}\right)=\min _{\alpha \in[0,+\infty)} k_{0}^{S H}(\alpha, \xi)=\sqrt{\xi^{2}+\frac{\pi^{2}}{4}} .
$$

In the case of the dispersion relation (8.2), similarly to (6.5), we have

$$
\Upsilon^{R L}\left(B_{\xi}\right)=\min _{\alpha \geqslant \xi} k_{0}^{R L}(\alpha, 0)
$$

Next, let us recall the asymptotic behaviour of $k_{0}^{R L}(\alpha, 0)$ near the lowest cut-off $k=\pi / 2$ (see Kaplunov et al. 1998). This gives the inequality

$$
k_{0}^{R L}(\alpha, 0)=\frac{\pi}{2}+\left(\frac{1}{\pi}+\frac{4 \chi}{\pi^{2} \tan \left(\frac{\pi}{2 \chi}\right)}\right) \alpha^{2}+O\left(\alpha^{4}\right) \geqslant \sqrt{\alpha^{2}+\frac{\pi^{2}}{4}}, \quad \alpha \rightarrow 0 .
$$

Similarly to section 6 , the curve $k_{0}^{R L}(\alpha, 0)$ intersects the curve $k=\sqrt{\alpha^{2}+\frac{\pi^{2}}{4}}$ only at the point $\alpha=0$. In combination with (8.5) and (8.6), this implies that $\Upsilon^{S H}\left(B_{\xi}\right) \leqslant \Upsilon^{R L}\left(B_{\xi}\right)$, and finally, we have

$$
\Upsilon\left(B_{\xi}\right)=\sqrt{\xi^{2}+\frac{\pi^{2}}{4}}
$$

A priori numerical experiments suggest that the test vector functions from the domain $d\left[a_{\xi}\right]$ should be chosen in the form

$$
\boldsymbol{u}^{t}\left(x_{1}, x_{2}\right)=\left(\begin{array}{c}
\cos \frac{\pi x_{2}}{2} f\left(x_{1}\right) \\
0 \\
i \cos \frac{\pi x_{2}}{2} h\left(x_{1}\right)
\end{array}\right)
$$

where $f\left(x_{1}\right), h\left(x_{1}\right) \in W_{2}^{2}\left(\mathbb{R}_{+}, \mathbb{R}\right)$. Then the variational functional (5.3) becomes

$$
k_{T}^{2}\left(B_{\xi}\right):=\frac{a_{\xi}\left[\boldsymbol{u}^{t}, \boldsymbol{u}^{t}\right]}{\left(\boldsymbol{u}^{t}, \boldsymbol{u}^{t}\right)}=\frac{\int_{0}^{\infty}\left(J_{1} f^{2}+J_{2} h^{2}+\chi^{2}\left(f^{\prime}\right)^{2}+\left(h^{\prime}\right)^{2}+2 \xi f h^{\prime}+J_{3} f^{\prime} h\right) d x_{1}}{\int_{0}^{\infty}\left(f^{2}+h^{2}\right) d x_{1}},
$$

where the coefficients $J_{1}, J_{2}$ and $J_{3}$ are given by

$$
J_{1}=\xi^{2}+\frac{\pi^{2}}{4}, J_{2}=\chi^{2} \xi^{2}+\frac{\pi^{2}}{4}, J_{3}=-2 \xi\left(\chi^{2}-2\right) .
$$

Using integration by parts, the denominator in (8.9) may be transformed to

$$
\begin{array}{r}
\int_{0}^{\infty}\left(\left(J_{1} f-\chi^{2} f^{\prime \prime}+\left(\xi-\frac{J_{3}}{2}\right) h^{\prime}\right) f+\left(J_{2} h-h^{\prime \prime}-\left(\xi-\frac{J_{3}}{2} f^{\prime}\right)\right) h\right) d x_{1}+ \\
-\left.\chi^{2} f f^{\prime}\right|_{x_{1}=0}-\left.h h^{\prime}\right|_{x_{1}=0}-\left.\left(\xi+\frac{J_{3}}{2}\right) f h\right|_{x_{1}=0}
\end{array}
$$


Therefore, the variational problem (8.9) may be reduced to the one-dimensional eigenvalue problem

$$
\breve{B}_{\xi}\left(\begin{array}{l}
f \\
h
\end{array}\right)=\left(\begin{array}{cc}
\xi^{2}+\frac{\pi^{2}}{4}-\chi^{2} \partial_{x_{1}}^{2} & \xi\left(\chi^{2}-1\right) \partial_{x_{1}} \\
-\xi\left(\chi^{2}-1\right) \partial_{x_{1}} & \chi^{2} \xi^{2}+\frac{\pi^{2}}{4}-\partial_{x_{1}}^{2}
\end{array}\right)\left(\begin{array}{l}
f \\
h
\end{array}\right)
$$

with

$$
\left.\left(\chi^{2} f^{\prime}+\xi\left(2-\chi^{2}\right) h\right)\right|_{x_{1}=0}=0,\left.\quad\left(h^{\prime}+\xi f\right)\right|_{x_{1}=0}=0
$$

(see Roitberg et al. (1998) for more detail). It can be easily verified that (8.11) corresponds to the quadratic form of the ordinary differential operator (8.12) with the boundary conditions (8.13).

To evaluate the minimum of $\sigma\left(\breve{B}_{\xi}\right)$ we consider the associated eigenvalue problem

$$
\breve{B}_{\xi} \boldsymbol{v}=\lambda \boldsymbol{v}, \quad \boldsymbol{v}=\left(\begin{array}{l}
f\left(x_{1}\right) \\
h\left(x_{1}\right)
\end{array}\right) \in D\left(\breve{B}_{\xi}\right) .
$$

The discrete eigenvalue is

$$
\lambda=k_{T}^{2}\left(B_{\xi}\right)=c_{R}^{2} \xi^{2}+\frac{\pi^{2}}{4}
$$

where the non-dimensionalised Rayleigh speed $c_{R}$ is a solution of the equation (A 4). As a result, the test vector function (8.8) becomes

$$
\boldsymbol{u}^{t}\left(x_{1}, x_{2}\right)=\cos \frac{\pi x_{2}}{2} \boldsymbol{r}_{\nu}\left(x_{1}, \xi\right)
$$

where $\boldsymbol{r}_{\nu}$ is the Rayleigh wave (A 2). Thus, we arrive at the estimate

$$
\min \sigma\left(B_{\xi}\right) \leqslant c_{R}^{2} \xi^{2}+\frac{\pi^{2}}{4}<\Upsilon^{2}\left(B_{\xi}\right), \quad \xi>0
$$

which proves that for $\nu<1 / 2$, there exists the lowest edge wave. In the twodimensional case $(\xi=0)$ the variational estimate (8.15) coincides with the lower bound of the continuous spectrum (8.7), i. e. $\Upsilon\left(B_{\xi}\right)=k_{t}\left(B_{\xi}\right)=\pi / 2$. For the latter the estimation can be carried out numerically, using the recipe given in section 7 . In Figure $2 \mathrm{~b}$ ) the computed value $k_{t}\left(B_{\xi}\right)$ is smaller than the lower bound of the continuous spectrum $\Upsilon\left(B_{\xi}\right)=\pi / 2$ for $\nu<1 / 2$.

\section{Numerical results}

In general, the eigenvalue problem (2.11) has a complex-valued spectrum corresponding to quasi-edge waves with non-localised components. It can be computed by exploiting the linear dependence of the three-dimensional Rayleigh-Lamb (3.8) and shear modes (3.10) similarly to the complex eigenvalues of a two-dimensional semi-infinite strip (see Zernov et al. 2006).

Dispersion curves of the fundamental edge wave in a plate with traction free faces are presented in Figures 3 a) and $3 \mathrm{~b}$ ). Figure 3 a) shows that the computed eigenvalue $k$ of the operator $A_{\xi}$ lies below the dotted line $\Upsilon\left(A_{\xi}\right)$, the numerically 


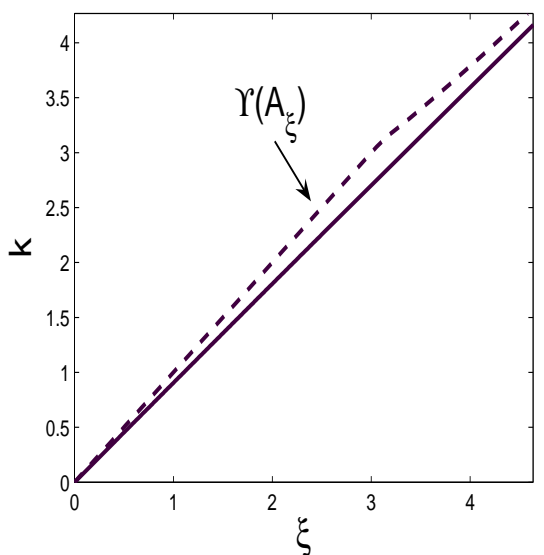

a)

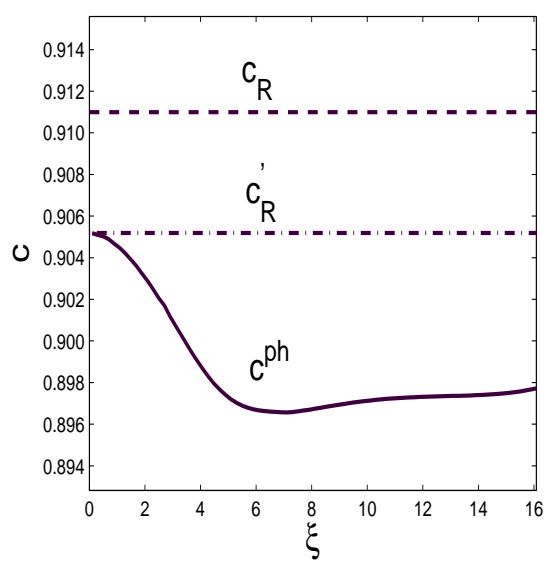

b)

Figure 3. The fundamental edge wave in a plate with traction free faces, $\nu=0.2$.

computed lower bound of the continuous spectrum (5.2). In Figure $3 \mathrm{~b}$ ) we show the phase velocity $c^{p h}=k / \xi$, the Rayleigh wave speed $c_{R}$ and, for comparison, the speed of the Rayleigh type wave $c_{R}^{\prime}$ in the two-dimensional theory of plate extension (e. g. Kaplunov et al. 1998). The latter is the solution of transcendental equation (A 4) with

$$
\chi^{2}=\frac{2(1+\nu)}{1-\nu^{2}}
$$

It is not surprising that in the long wavelength limit $(\xi<<1), c_{R}^{\prime}$ proves a highly accurate approximation to the speed of the fundamental edge wave.

Figures 4 a) and 4 b) dysplay the dispersion curves of the first order edge wave in a plate with traction free faces. Here $k$ is a complex valued quantity. The corresponding cut-off frequencies $k(0)$ are the eigenfrequencies of the two-dimensional semi-infinite strip (Zernov et al. 2006). They are real for $\nu=0$ and $\nu \approx 0.22475$. For $\nu=0$, and only for this value, there exists the whole region of real eigenvalues (see also the discussion in section 8 ). In Figure $4 \mathrm{~b}$ ) this region is $0 \leqslant \xi \leqslant 1.1585$. For $\xi>1.1585$, the first Rayleigh-Lamb harmonics propagates along $x_{1}$ and the corresponding eigenfrequencies $k$ are shifted to the complex domain. By contrast for $\nu \approx 0.22475$ and $\xi>0$ we have $\operatorname{Im} k(\xi)<0$ (see Figure 5 ). This is due to the coupling with the fundamental shear mode that propagates along $x_{1}$.

The dispersion curves of the lowest edge wave that propagates in a plate with fixed faces are shown in Figures 6 a) and $6 \mathrm{~b}$ ). Figure 6 a) confirms that such a curve lies below the dotted line $k_{t}\left(B_{\xi}\right)$ as given by $(8.15)$.

Figure $6 \mathrm{~b}$ ) presents the comparison of the computed phase velocity $c^{p h}$ with the variational estimate $c_{t}=k_{t} / \xi$ as given by (8.15).

\section{Concluding remarks}

We have generilised to 3D the numerical procedure for calculating edge modes in a 2D semi-infinite strip with traction free boundary (Zernov et al. 2006). We have 


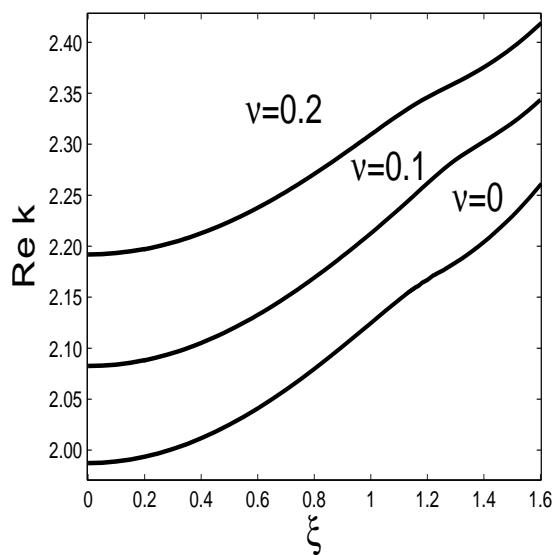

a)

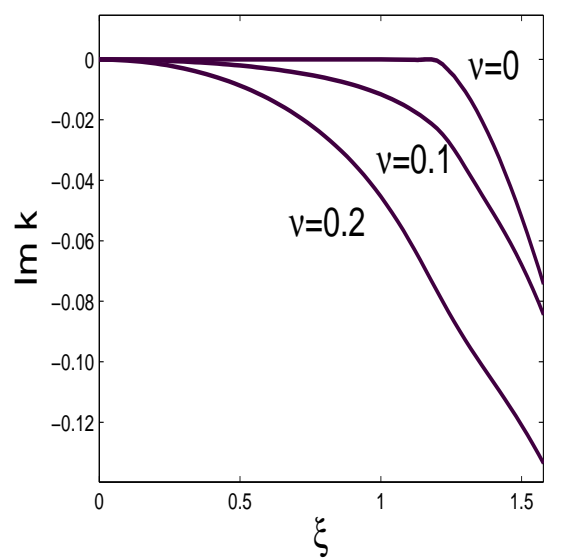

b)

Figure 4. First order edge waves in a plate with traction free faces.

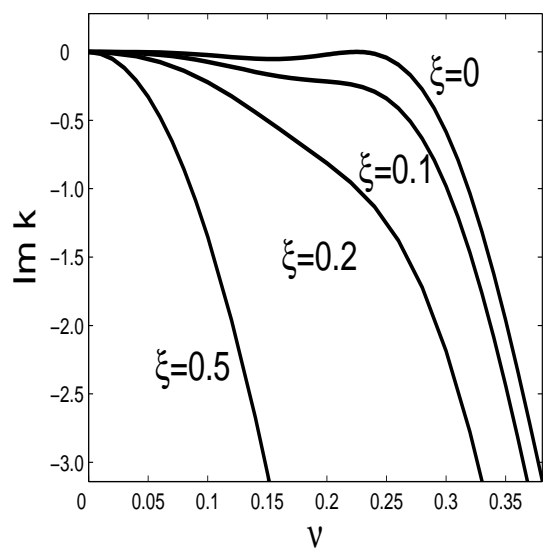

Figure 5. The effect of the Poisson ratio.

proven that for any $\nu$, in a plate with traction free boundaries, there exists a fundamental edge. We have found an upper variational estimate of the fundamental dispersion curve. For $\nu=0$, we have shown that the fundamental edge wave coincides with the Rayleigh wave and therefore, the solution may be obtained explicitly. Note that in the $2 \mathrm{D}$ case there is no edge mode corresponding to the fundamental edge wave.

We have also addressed the question of orthogonality conditions between various modes. In the 2D case such conditions have been established for $\nu=0$ and the socalled Lamé frequency (Zernov et al. 2006). Here we have shown that for $\nu=0$ the orthogonality conditions may be generalised, whereas for the Lamé frequency no orthogonality conditions exist. As a consequence, for $\nu=0$, the fist harmonic edge wave is present in a frequency band and its cut-off frequency coincides with the 


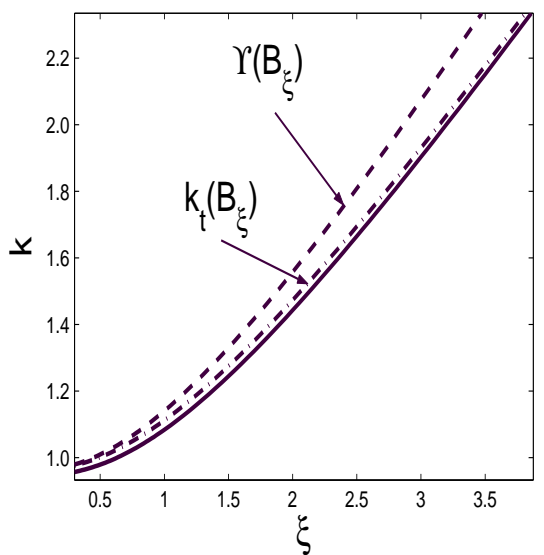

a)

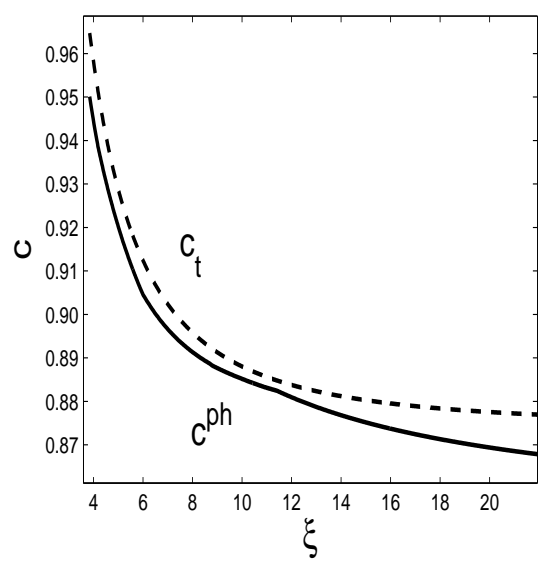

b)

Figure 6. The lowest order edge waves in a plate with fixed faces, $\nu=0.2$.

real eigenvalue for a $2 \mathrm{D}$ semi-infinite strip. For the Lamé frequency, the eigenvalue of the first harmonic wave is complex, i.e. there is no wave localised near the edge.

Finally we have analysed edge modes and edge waves in a plate with fixed faces. Obviously, there can be no fundamental edge wave in such a plate. However, we have shown that for any $\nu \in[0,0.5)$, there exists the first harmonic edge wave. Its cut-off frequencies correspond to the edge modes of a $2 \mathrm{D}$ semi-infinite strip with fixed faces. In contrast to the 2D strip with traction free faces, these eigenvalues are real.

\section{Appendix A. The Rayleigh wave}

The classical surface Rayleigh wave can be presented in the form

$$
\begin{gathered}
\boldsymbol{U}\left(x_{1}, x_{2}, x_{3}\right)=\boldsymbol{r}_{\nu}\left(x_{1}, \xi\right) e^{\mathrm{i} \xi x_{3}}, \\
\boldsymbol{r}_{\nu}\left(x_{1}, \xi\right)=\left(\begin{array}{c}
2 \\
0 \\
2 \mathrm{i} \Lambda_{L}
\end{array}\right) e^{-\xi \Lambda_{L} x_{1}}-\left(\begin{array}{c}
\Lambda_{T}^{2}+1 \\
0 \\
\mathrm{i} \frac{\Lambda_{T}^{2}+1}{\Lambda_{T}}
\end{array}\right) e^{-\xi \Lambda_{T} x_{1}},
\end{gathered}
$$

with

$$
\Lambda_{L}=\sqrt{1-\frac{c_{R}^{2}}{\chi^{2}}}>0, \quad \Lambda_{T}=\sqrt{1-c_{R}^{2}}>0,
$$

where the Rayleigh speed $c_{R}\left(c_{R}<1\right)$ satisfies the transcendental equation

$$
\left(2-c_{R}\right)^{2}-4 \sqrt{1-c_{R}^{2}} \sqrt{1-\frac{c_{R}^{2}}{\chi^{2}}}=0 .
$$

It can be easily verified that $(A 1)$ is a solution the equations of motion $(2.1)$ with $k=c_{R} \xi$, i. e. of the equation

$$
A_{\xi} \boldsymbol{r}_{\nu}\left(x_{1}, \xi\right)=c_{R}^{2} \xi^{2} \boldsymbol{r}_{\nu}\left(x_{1}, \xi\right)
$$




\section{References}

Adams, S. D. M., Craster, R. V. \& Williams, D. P. (2007) Rayleigh waves guided by topography. Proc. R. Soc. A., 463, 531-550.

Birman, M. S. \& Solomjak, M. Z. (1986) Spectral theory of self-adjoint operators in Hilbert space. Reidel, Dordrecht.

Davies, E. B. (1995) Spectral theory and differential operators. University Press, Cambridge.

Förster, C., WeidL, T. (2006) Trapped modes for an elastic strip with perturbation of the material properties. Q. J. Mech. Appl. Math., 59, 399-418.

Gobert, J. (1962) Une inegalite fondamentale de la theorie de la l'elasticite. Bull.Soc.Royale Sciences Liege., 31, 182-191.

Kaplunov, J. D., Prikazchikov, D. A. \& Rogerson, G. A. (2005) On three dimesoinal edge waves in pre-stressed incompressible elastic solids. J. Acoust. Soc. Am., 118(5), 2975-2983.

Kaplunov, J. D., Kossovich, L. Y. \& Nolde, E. V. (1998) Dynamics of thin walled elastic bodies. New York: Academic Press.

Kaplunov, J. D., Kossovich, L. Y. \& Wilde, M. V. (2000) Free localised vibrations of a semi-infinite cylindrical shell. J. Acoust. Soc. Am., 107, 13831393.

Kaplunov, J. D., Wilde, M. V. (2000) Edge and interfacial vibrations in elastic shells of revolution. ZAMP, 51, 530-549.

Konenkov, Yu. K. (1960) A Rayleigh-type flexural wave. Sov. Phis. Acoust., 6, $122-123$.

Love, A. E. H.,(1944) A Treatise on the Mathematical Theory of Elasticity. New York: Dover Publications.

Miklowitz, J. (1978) The theory of elastic waves and waveguides. North Holland.

Pagneux, V.(2006) Revisiting the edge resonance for Lamb waves in a semi-infinite plate. J. Acoust. Soc. Am., 120(2), 649-656.

Postnova, J., Craster, R. V. (2006) Trapped modes in topographically varying elastic waveguides. Wave Motion, 44, pp. 205-221.

Roitberg, I., Vassiliev, D. \& Weidl, T. (1998) Edge resonance in an elastic semi-strip. Q. J. Mech. Appl. Math., 51, 1-13.

Fu, Y. B.(2003) Existence and uniqueness of edge waves in a generally anisotropic elastic plate. Q. Jl. Mech. Appl. Math., 56, 605-616.

Zernov, V., Pichugin, A. V. \& Kaplunov, J. (2006) Eigenvalue of a semiinfinite elastic strip. Proc. R. Soc. A., 462, 1255-1270.

Zernov, V. (2006) PhD Thesis. Brunel University. 